\title{
The Application of "Big Data Analysis + Hierarchical Medical" Model in the Context of the COVID-19
}

\author{
Xingyu Yang ${ }^{1, *}$, Tianyu Yan $^{1}$, Zelong Huang ${ }^{1}$, Xiaofang Zhang ${ }^{2}$, Yuchen Zhao ${ }^{3}$, and Zihao $\mathrm{Jin}^{4}$ \\ ${ }^{1}$ Qingdao University of Technology, School of Management Engineering, 266525 Qingdao, China \\ ${ }^{2}$ Qingdao University of Technology, Higher Vocational and Technical College, 266525 Qingdao, China \\ ${ }^{3}$ Qingdao University of Technology, Business School, 266525 Qingdao, China \\ ${ }^{4}$ Qingdao University of Technology, School of Humanities and Foreign Languages 266525 Qingdao, China
}

\begin{abstract}
The COVID-19 epidemic has swept the world, causing serious impact and influence on economic development and residents' life in countries all over the world. This paper takes China as an example, further analyses the characteristics of China's hierarchical medical model based on the international hierarchical medical research planning, and proposes the application of "big data analysis + hierarchical medical" model for the new coronavirus epidemic and other public health emergencies based on the advantages of big data application to solve public health crises, in order to provide a reference for the planning of hierarchical medical system during the epidemic. It is expected to provide reference for the planning of hierarchical medical and health system during the epidemic, which is an innovative attempt of the medical industry.
\end{abstract}

\section{Introduction}

In early December 2019, the first case of the COVID-19 infection within China appeared in Wuhan, Hubei Province, and since then, along with the horrific spreading power of the novel coronavirus, various provinces and regions in China began emergency work deployment arrangements for the war on the epidemic, while governments around the country also introduced a series of relevant policies. This new coronavirus epidemic reflects to some extent the peculiarities of the development of China's urban medical level system. The main reason for the spread of the epidemic was the lack of vaccines for the epidemic virus and the inability to distinguish between infected and uninfected people, which led to a massive cross-infection of the virus using the time lag between diagnosis and detection. Therefore, in order to better cope with the epidemic control, cities should face a series of problems such as low number of hospitals, vacant beds, and uneven service levels at the grassroots community level, and implement a "tiered medical care" system to promote a reasonable distribution of medical resources to ensure that localities can handle emergencies with ease again.

On the other hand, the advent of big data in the world has provided an opportunity to combine big data analysis with statistical application technology, which can be applied to the existing hierarchical medical system in China to solve public health emergencies efficiently, accurately and conveniently.

This paper analyses China's current urban health care system from a health care perspective, distils the advantages of the international health service system, and proposes a stable and reliable "big data analysis + hierarchical medical " model to combat epidemics based on the application of big data analysis during the COVID-19 and the layout of China's epidemic prevention plan, in order to provide some reference for the planning of the tiered medical care system during the epidemic.

\section{Analysis of the current situation of china's hierarchical medical system}

According to the survey data, the current situation of medical service supply in China diverges from the system reform of hierarchical medical - the hierarchical medical care model is hollowed out internally. The number of visits to tertiary hospitals is far greater than the number of visits to primary care institutions; the top advanced medical resources are converging on large hospitals, and the number of patients received in their outpatient and inpatient departments far exceeds the supply, which is a large-scale trend, and the phenomenon of "siphoning" is obvious. Primary care institutions are not utilizing many medical equipment resources because there are fewer patients to be seen. Because of the low number of patients in primary care, many medical equipment resources are not utilized, and the function of treating common diseases is weakening. The problem is mainly twofold:

(1) The problem of "two major and one public" in public hospitals. Since the implementation of the "new medical reform" policy, although county-level public

\footnotetext{
" Corresponding author: 785746806@qq.com
} 
hospitals and urban public hospitals have been supported by national policies and have carried out corresponding reforms, the hospital structure model for public hospitals has not reached the ideal state, mainly reflected in the "two major and one public" public hospitals. "However, the hospital structure model is not ideal for public hospitals. This defect is the main cause of the uneven distribution of medical resources, also known as the "inverted triangle", mainly in the main medical services health resources concentrated in large and medium-sized cities, and more part of the concentration in large public hospitals. However, from the perspective of demand for medical resources, the demand for medical services at the grassroots level accounts for a large proportion, showing a "positive triangle" structure. The inverted triangle structure of medical resource supply and the positive triangle structure of medical service demand solidify the inertia of residents' medical behavior, intensifying the difficulty of patients' access to medical care and increasing the medical costs borne by patients.

(2) Imperfect system of medical treatment in different places. In the scope of social health insurance, the term "off-site" generally refers to a domestic area other than the insured area. The term "medical treatment" refers to the medical treatment of a participant. Off-site referral for medical treatment greatly satisfies patients' demand for high medical resources and protects people's life and property. However, the current system lacks a precise referral mechanism to guide patients, resulting in disorderly medical treatment, which ultimately results in a large number of patients seeking higher-level medical treatment in large and mediumsized cities. This pattern of disorderly off-site medical treatment not only increases the cost burden of patients, but also to some extent weakens the capacity of primary medical institutions.

\section{International sub-medical model advantages to learn from}

\subsection{Health system-based hierarchical medical model}

The National Health Service (NHS), or the British National Health Service, is a system that adheres to the principle of selective relief for the poor and is dedicated to the task of ensuring publicly funded health care for all in the UK [3]. The health care system is based on primary care centers, with hospitals as a support. Referrals are made in different levels of direction according to the risk factor of the patient, who would otherwise not have direct access to the hospital.

\subsection{Commercial insurance-based hierarchical health model}

The U.S. tiered health care system is based on commercial insurance, unlike the Canadian system, which is based on universal health insurance. Social health insurance in the U.S. tiered health care system is designed to cover the elderly and vulnerable groups, while for those with stable jobs they need to provide fees to commercial insurance providers to obtain health insurance. This model has been established mainly by the advanced medical equipment and high level of general practitioners in U.S. primary care hospitals. A significant number of U.S. primary care hospitals are able to reach the level of secondary and even tertiary care hospitals.

\subsection{Analysis of advantages}

For any country's medical service system, the basic concept of graded medical care depends on whether selfcare can solve the problem, and if it cannot, then it needs to seek treatment from primary care doctors, and if the primary care institutions cannot handle it, then it needs to be referred to large medical and health institutions to meet the different needs of medical services at different levels.

We can see from the mainstream hierarchical medical model in the United Kingdom and the United States that both countries have adopted a high degree of attention to primary care, combined with China's own national characteristics, so we can draw three lessons from it: 1. 2 . to establish a reasonable grading payment system. The graded payment should not only be planned for different types of medical services in each region, but also for different attributes of medical services such as health management - chronic disease management - outpatient services - inpatient medical care - rehabilitation treatment - long-term care - hospice care. A combination of payment methods can be adopted, and the different functional positioning of different medical service institutions should be taken into account when issuing policies to encourage and support them. 3. Medical and health care should increase the investment, according to the data of the Health and Welfare Commission, in 2018, the per capita cost of medical and health care expenditure in China was 4237 yuan, the per capita cost of expenditure in the United Kingdom and the United States was 68,544.9975 and 26,525.485 yuan, and the gap is still large compared with developed countries, and the total medical and health care expenditure in the United Kingdom and the United States accounted for $16.9 \%$ and $9.8 \%$ in 2018, and China Health care expenditure only accounted for $6.43 \%$ of GDP.

\section{Application of "big data analysis + hierarchical medical care" model}

The main reason why the graded medical system is limited in China is that the primary medical institutions cannot meet the needs of patients due to medical resources and medical care level, and the main problem is still at the grassroots level. And big data analysis technology needs to collect real data in a short period of time in a wide range, and it needs to be more relevant to the grassroots. The combination of the two forms the "big data analysis + graded medical care" model, which realizes the interface between primary medical 
institutions and the grassroots. Especially in the current state of epidemic transmission, the "big data analysis + graded medical care" model can take advantage of the large number of primary care institutions and their proximity to the masses to obtain a large amount of accurate epidemic transmission data, and also solve the shortcomings of China's graded medical care system in the process, and the two complement each other. The specific application of both can be divided into two segments.

\subsection{Epidemic prevention and control monitoring link application}

Before the outbreak of an epidemic and at the early stage of infection, there is often a certain period of stability, during which the number of infected people is small and the scope of infection is relatively small, and this is the best time for preventive intervention, and if it is detected at this time, it can be controlled. However, in actual transmission, medical institutions and transmitters pay little attention to it, which leads to the incubation of the virus and eventually to widespread transmission, so epidemic prevention and control surveillance is the basis for controlling the epidemic. The current outbreak exposed the deficiencies in outbreak prevention and control surveillance in China, as according to the survey new crown pneumonia infections had already appeared in December 2019 but did not receive appropriate attention. However, the use of big data capture information technology combined with the hierarchical medical system can be a good way to avoid the recurrence of the situation and improve the ability to detect epidemic prevention and control.

(1) Combination of primary medical institutions and big data front-end. The combination of primary medical institutions and the big data front-end constitutes a source of information for the original data capture, because primary medical institutions are closer to a larger proportion of the patient population by virtue of their convenience, and realize the mutual traffic of disease information with the grassroots. The big data system is responsible for including all patient information in the personal information database, and if suspected symptoms or other unmanageable symptoms are found, the primary care organization will evaluate the patient's disease information according to the actual international standard epidemic infection status, which can be referred to the "International Disease Outbreak Response System Dorscon" standard The epidemic is graded into four levels: green, yellow, orange, and red, and once it is found to exceed the specified range, the big data processing system immediately disseminates the information to the next processing center and causes an early warning. The front end achieves transparency, openness, and efficiency of all epidemic patient disease information, avoiding the shortcomings of hidden data information and untimely dissemination of epidemic information, and truly playing the role of primary care institutions.

(2) Medium and large hospitals combined with big data backstage. Because of the complete medical equipment and high level of medical care, medium and large hospitals combine the big data backend with them to achieve a strong combination. Because the big data backstage needs to process a large amount of data and summarize and classify all the data coming from primary medical institutions, it needs a system with better equipment and hardware and more stable performance to support. One of the more prominent points in the background is the establishment of a "network epidemic monitoring system", which can be used to integrate and filter data from various social networks and media, and determine whether there is currently negative information about the epidemic spreading or hiding information about infected patients according to the established sensitive word database system, realizing the use of network The epidemic is monitored using network data. The combination of the front-end and the back-end really realizes the epidemic prevention and control monitoring. 
Front-end: Big data epidemic information collection and release system

\begin{tabular}{|c|c|}
\hline Collect and process basic patient information & Hierarchy of warning messages sent \\
\hline Hierarchy of warning messages sent & Automatic release of warning information \\
\hline $\begin{array}{c}\text { 1.Red、Orange } \rightarrow \text { The state administrative } \\
\text { department for health }\end{array}$ & $\begin{array}{l}\text { 1.Red、 Orange } \rightarrow \text { The official website of the } \\
\text { National Health Department }\end{array}$ \\
\hline $\begin{array}{l}\text { 2.Yellow、Green } \rightarrow \text { Provincial health } \\
\text { administrative departments }\end{array}$ & $\begin{array}{l}\text { 2.Yellow } \text { Green } \rightarrow \text { The official website of } \\
\text { provincial health departments }\end{array}$ \\
\hline
\end{tabular}

Background: Big data epidemic monitoring and processing system

\begin{tabular}{|c|c|c|}
\hline National data & Social Data & Network Data \\
\hline \multicolumn{3}{|c|}{ 1.Data screening, data classification } \\
2.Standard typed data \\
3.Data desensitization, data filtering \\
4.Network epidemic detection system \\
\hline
\end{tabular}

Figure 1. Big data prevention and control monitoring platform architecture

\subsection{Application of medical resource allocation by region during an epidemic}

The big data platform can also be applied to medical resource allocation because one of the serious problems in China's current hierarchical medical system is the uneven distribution of medical hospitals, and under the stimulus of the current epidemic spread, medical resource scheduling has become a problem that must be solved. According to the traditional method, medical resources need to be registered and recorded by all parties, and if one party in the whole chain does not communicate in time, then there will be a lag, which seriously affects the process of fighting the spread of the epidemic. However, by establishing a resource dispatching model with big data technology as the core, we can coordinate and optimize all resources, so that medical resources can accurately and timely meet the needs of each region for epidemic treatment. The model consists of two aspects.

(1) The big data technology platform is a comprehensive platform that can combine hospitals, patients, logistics and other aspects, and the information on the demand and supply of medical hospitals on this platform should be transparent and open to eliminate the information lag. The big data technology platform can transmit information to the resource supplier according to the needs of all parties, so that the supplier can complete the preparation of medical resources in advance and distribute them according to the demand, and use the Internet of Things technology in the transportation of resources to accurately locate resources and transmit GPS information, and establish a relevant model according to the real-time road conditions to arrive at the optimal route plan to the destination, so as to provide resources during the epidemic Distribution and delivery.

(2) The big data platform can data predict the demand for resources to ensure adequate medical resources. The big data platform should not only achieve the use of current data to complete hospital dispatch allocation and plan the optimal route, but also predict the possible situations to be prepared in case of emergency and prevent casualties caused by medical resource vacancies. The essence is to realize the control of medical resources and improve the uneven distribution of graded medical resources based on the known amount of data such as the amount of medical resources distribution demand, the epidemic growth status, the number of people admitted to hospitals at different regional levels, and the remaining amount of medical resources supply, and then introduce the values of variables such as time and cost to substitute into the resource demand model of the system. 


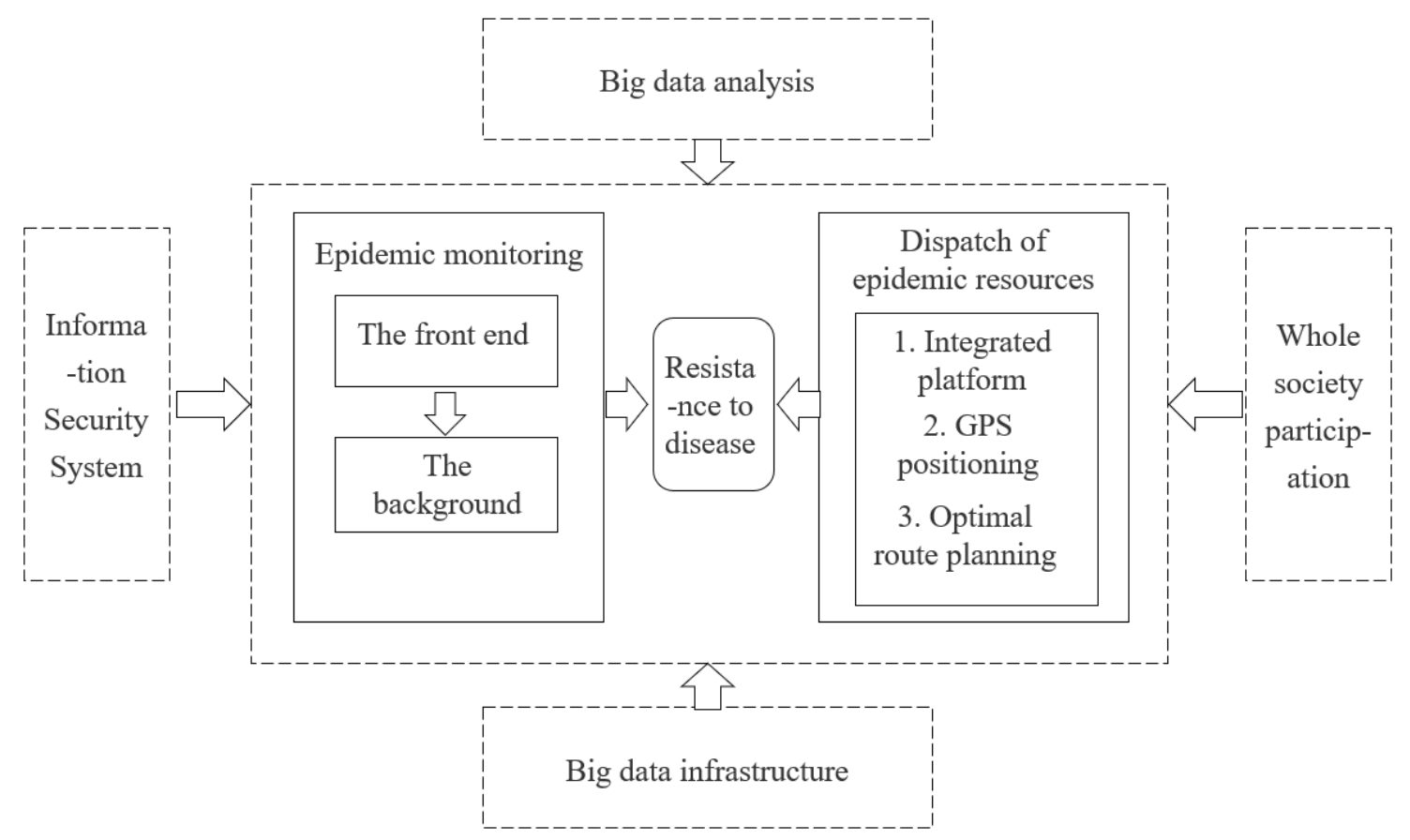

Figure 2. "Big Data Analysis + hierarchical Medical " model application diagram

\section{Conclusion}

It is undeniable that the sudden arrival of the epidemic disrupted the normal financial and economic development and normalization of life, but on the other hand, the epidemic also promoted the current medical system and the formation of the "big data analysis + tiered medical care" model. Although China is now in the post-epidemic era, many countries around the world are still in deep water, and some mutated viruses still exist, so countries still need to promote the development of medical standards, improve the model of graded medical system, improve the medical security mechanism, and realize the accurate control of all stages of public health emergencies such as epidemics on the basis of the advantages of big data analysis, so as to meet the diversified and multi-level medical needs and health needs of the people, and escort for human health.

\section{References}

1. X. Ouyang et al., "Dual-Sampling Attention Network for Diagnosis of COVID-19 From Community Acquired Pneumonia," in IEEE Transactions on Medical Imaging, vol. 39, no. 8, pp. 2595-2605, Aug. 2020.

2. R. Han, Z. Liu, C. L. p. Chen, L. Xu and G. Peng, "Mortality prediction for COVID-19 patients via Broad Learning System," 2020 7th International Conference on Information, Cybernetics, and Computational Social Systems (ICCSS), Guangzhou, China, 2020, pp. 837-842.

3. A. K. Siddhu, A. Kumar and S. Kundu, "Review Paper for Detection of COVID-19 from Medical Images and/ or Symptoms of Patient using Machine
Learning Approaches," 2020 9th International Conference System Modeling and Advancement in Research Trends (SMART), Moradabad, India, 2020, pp. 39-44.

4. Y. -H. Wu et al., "JCS: An Explainable COVID-19 Diagnosis System by Joint Classification and Segmentation," in IEEE Transactions on Image Processing, vol. 30, pp. 3113-3126, 2021.

5. L. Zhou et al., "A Rapid, Accurate and MachineAgnostic Segmentation and Quantification Method for CT-Based COVID-19 Diagnosis," in IEEE Transactions on Medical Imaging, vol. 39, no. 8, pp. 2638-2652, Aug. 2020.

6. H. Ren, J. Shen, X. Tang and T. Feng, "5G Healthcare Applications In COVID-19 Prevention And Control," 2020 ITU Kaleidoscope: IndustryDriven Digital Transformation (ITU K), Ha Noi, Vietnam, 2020, pp. 1-4.

7. R. Y. Wang, T. Q. Guo, L. G. Li, J. Y. Jiao and L. Y. Wang, "Predictions of COVID-19 Infection Severity Based on Co-associations between the SNPs of Comorbid Diseases and COVID-19 through Machine Learning of Genetic Data," 2020 IEEE 8th International Conference on Computer Science and Network Technology (ICCSNT), Dalian, China, 2020, pp. 92-96.

8. M. Chen and Y. Zhao, "Discussion on Coordination Problems and Strategies of Medical and Health Systems in Health Emergency Treatment : A case study of COVID-19 emergency treatment in Wuhan city," 2020 International Conference on Public Health and Data Science (ICPHDS), Guangzhou, China, 2020, pp. 398-401. 